\title{
Modelos de Riesgo de Crédito: Aplicación Práctica a un Modelo de Refinanciación de Hipotecas
}

\author{
CARO BARRERA, JosÉ RAFAEL \\ Departamento de Estadística, Econometría, Investigación Operativa, \\ Organización de Empresas y Economía Aplicada \\ Universidad de Córdoba \\ Correo electrónico: z52cabaj@uco.es
}

\begin{abstract}
RESUMEN
Ante un hipotético, pero cada vez más real, caso de riesgo de impago de una hipoteca o ante una caída en los tipos de interés, un asunto importante que se plantea el prestatario es el de la posibilidad de minimizar ese riesgo mediante la selección de la mejor opción de refinanciación. En el presente trabajo se presenta un modelo de refinanciación de hipotecas desarrollando un método de programación puramente cuantitativa, con una simulación basada en un algoritmo creado especialmente para este caso y que puede ser útil para deudores hipotecarios. Así, se comienza explicando la base teórica sobre la que se asienta la investigación, para pasar a desarrollar el problema, continuando con su implantación. Finalmente, se analizan los resultados y se comentan las conclusiones más relevantes.
\end{abstract}

Palabras clave: riesgo de crédito, optimización, programación dinámica, tipo de interés, refinanciación hipotecaria, valores respaldados por hipotecas, Basilea III.

Clasificación JEL: C02; C61; C63.

MSC2010: 49L20; 49N20; 60J25; 91G40. 


\title{
Credit Risk Models: Practical Application to a Mortgage Refinancing Model
}

\begin{abstract}
Facing an hypothetical, but increasingly, case of default risk on a mortgage or a fall in interest rates, an important issue raised by the borrower is the possibility of minimizing that risk by selecting the best refinancing option. In this paper, a mortgage refinancing model is presented, developing a purely quantitative programming method with a simulation based on an algorithm created especially for this case and that can be useful for mortgage debtors. Thus, we begin by explaining the theoretical basis on which the research is based, to proceed to develop the problem, continuing with its implementation. Finally, the results are analyzed and the most relevant conclusions are commented.
\end{abstract}

Keywords: credit risk, dynamic optimization, dynamic programming, interest rate, mortgage refinancing, mortgaged backed securities, Basel III.

JEL classification: C02; C61; C63.

MSC2010: 49L20; 49N20; 60J25; 91G40.

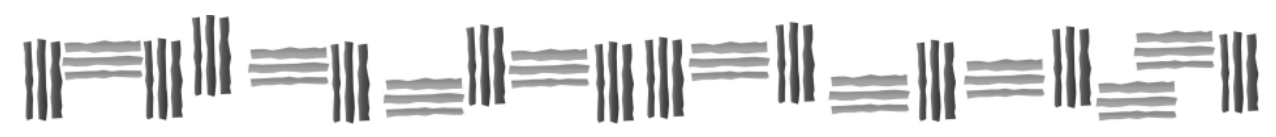




\section{Introducción.}

Una hipoteca es un préstamo garantizado por bienes raíces. El total del préstamo, llamado principal, se paga a lo largo de un horizonte temporal mediante cuotas. Estos pagos son, por lo general, calculados prorata temporis, lo que significa que cada flujo de caja se divide en dos componentes: un primer componente representa una parte del saldo pendiente, un segundo componente es el interés devengado desde el último pago. Este esquema garantiza que el préstamo es totalmente pagado al final del contrato. En la mayoría de las hipotecas residenciales, los préstamos ofrecidos a los inversores particulares son amortizados a través del esquema "francés" de amortización, que consiste en cuotas constantes, por lo general pagaderas mensualmente.

Mención especial merece el caso del mercado estadounidense, donde la amplia mayoría de las hipotecas son recogidas en grupos de préstamos gestionados por agencias patrocinadas por el gobierno. Estas instituciones emiten pagarés de titulización respaldados por los flujos de efectivo generados por un grupo específico de las hipotecas y venden este tipo de activos, conocidos como valores respaldados por hipotecas (Mortgage Backed Securities, MBS en sus siglas en inglés), a inversores privados, generalmente grandes fondos de inversión, tanto directamente como a través de distribuidores. Es importante destacar que gran parte de la crisis que afectó a los EEUU en 2008 fue provocada por culpa de este producto financiero.

La intención de este trabajo es encontrar una política de refinanciación óptima visto desde la óptica del deudor hipotecario, cuestión que autores como Pliska (2006) aborda trabajando con el comportamiento del deudor hipotecario en tiempo discreto y describiendo este comportamiento como un proceso de intensidad adecuada con tasas de hipoteca exógenas, así, el valor del contrato se deduce en una forma explícita y que se interpreta como el principal más el valor de un cierto swap. Longstaff (2005) en cambio tiene como objetivo minimizar los costes de la hipoteca con una estrategia de refinanciación óptima recursiva y examinando las implicaciones de esta estrategia en la evaluación de los MBS.

En el caso que se ha desarrollado, hemos seguido las bases teóricas introducidas por Roncoroni y Moro (2006), donde se considera el problema de la refinanciación de la hipoteca, tratando de mostrar la forma de determinar el estado óptimo de prepago minimizando el valor de la posición de la hipoteca y eligiendo el tiempo de la misma para ser financiada. Así, los autores anteriormente mencionados, agregan una restricción en el número de refinanciaciones.

Esta función contempla la posibilidad de que el banco (prestamista) le da al prestatario la opción de refinanciar la hipoteca internamente a menor costo adicional que el que se presenta al pagar el principal y entrar en una nueva hipoteca con otro prestamista (por ejemplo, los costes de transacción y las variaciones diferenciales de crédito).

De este modo, se asume que la refinanciación está sujeta a pequeñas comisiones y costes de transacción y se centran en que las condiciones de refinanciación son en las mejores condiciones de mercado para el prestatario. La inclusión de elementos exógenos que conducen la política de refinanciación no plantea ningún problema particular en la propuesta establecida. Es suficiente para permitir que el horizonte de tiempo dependerá de la ocurrencia de eventos aleatorios que desencadenen la expiración del contrato. El número $N$ de oportunidades de refinanciación en el horizonte temporal está fijado desde el principio. En cada momento, el deudor hipotecario establece la tasa de la deuda tanto a la tasa actual como a la tasa de interés variable nuevamente disponible. Sobre la vida del contrato, esta opción puede ser ejercida $N$ veces como máximo.

Desde un punto de vista financiero, el problema de decisión del deudor hipotecario toma la forma de una opción múltiple compuesta americana. Esto permite el uso de métodos de programación dinámica estocástica para hacer frente a la determinación de la política de prepago óptima definida como la que minimiza el valor del contrato. 
El trabajo que presentamos está organizado como sigue. En el punto 2 se plantea el problema y se describe la función del contrato de hipoteca de tipo fijo y tipo variable bajo opciones de refinanciación limitadas, basado en Roncoroni y Fusai (2008). La sección 3 detalla la metodología de la solución propuesta, el algoritmo creado y su implantación y extiende el análisis del caso incluyendo costes de transacción. El apartado 4 ilustra los resultados empíricos obtenidos mediante la realización de experimentos en escenarios alternativos. Finalmente, el último punto, presenta las principales conclusiones, comentarios y últimos avances y aportaciones en este campo.

\section{Planteamiento del problema y solución.}

\subsection{Hipoteca con tipo fijo.}

Se considera un horizonte temporal finito $T_{0, T}:=(0,1, \ldots, T)$. En el momento 0 , un individuo toma prestado $1 €$. Esto genera un saldo a pagar $B(0)=1$. El prestatario está obligado a pagar esta cantidad junto con los intereses más allá del horizonte de acuerdo a un esquema de entrega constante. Para cada período $[t-1, t]$ se calcula un interés $I(t-1)$, sobre el saldo pendiente $B(t-1)$ a una tasa igual a $r(t-1)$. El riesgo vivo $B^{\prime}(t-1)$ del deudor en el período $[t-1, t]$, se define por el interés $I(t-1)$ más el equilibrio de pie $B(t-1)$. Al final del período, es decir, el tiempo $t$, el prestatario paga una fracción $f(t)$, la cual está escrita en función de la exposición $B^{\prime}(t-1)$.

Este número se calcula como un porcentaje del tiempo de vida residual del contrato, es decir, $f(t)=1 / T-(t-1))$. Se denota este pago por $P(t-1)$. La dependencia explícita en el día de comienzo $t-1$ del período, subraya que este importe se fija en este momento, aunque saldado al final del mismo. Después de realizar este pago, el nuevo saldo en circulación para el deudor se convierte en $B(t)=B^{\prime}(t-1)-P(t-1)$.

La tabla 1 resume los pasos a seguir en este plan de pago. Está claro que el nuevo saldo depende del saldo inicial y del proceso de la deuda a una tasa $r=(r(t), t=0, \ldots, T-1)$. Puesto que por hipótesis la posición inicial de la deuda es $B(0)=1$, la única variable exógena es la tasa $r$.

Tabla 1. Esquema de plan de pago.

\begin{tabular}{lll}
\hline \multicolumn{1}{c}{ Cantidad } & Notación & Fórmula \\
\hline Deuda pendiente en $t-1$ & $B(t-1)$ & Dada por inducción \\
Tasa de deuda para el período $[t-1, t]$ & $r(t-1)$ & Aleatoria \\
Intereses acumulados en $[t-1, t]$ & $I(t-1)$ & $r(t-1) \cdot B(t-1)$ \\
Exposición pendiente en $[t-1, t]$ & $B^{\prime}(t-1)$ & $I(t-1)+B(t-1)$ \\
Coeficiente de entrega constante & $f(t)$ & $1 /(T-(t-1))$ \\
Pago al prestamista en $t$ & $P(t-1)$ & $f(t) \cdot B^{\prime}(t-1)$ \\
Deuda pendiente en $t$ & $B(t)$ & $B^{\prime}(t-1)-P(t-1)$ \\
\hline
\end{tabular}

Fuente: Elaboración propia.

Hay que tener en cuenta que el capital prestado es totalmente pagado al final del horizonte temporal, es decir, $B(T)=0$. Esto se demuestra mostrando que el último pago $P(T-1)$ coincide con el equilibrio permanente en $T-1$ :

$$
\begin{aligned}
P(T-1) & =B^{\prime}(T-1) /(T-(T-1)) \\
& =B^{\prime}(T-1)
\end{aligned}
$$


El coste de ir asociado un proceso de endeudamiento a una tasa $r$ dada se define como la suma de todos los flujos de efectivo derivados del régimen de pago en el esquema descrito anteriormente, es decir, $\Sigma_{t=0}^{T-1} P(t)$. A continuación se describe el proceso de la tasa $r$.

\subsection{Hipoteca con tipo variable.}

Se asume que el mercado da una tasa o tipo de interés $R$ y se supone que este número sigue un proceso de Markov temporal homogéneo $R=(R(t), t=0, \ldots, T)$ con espacios de estado finitos $\mathcal{S}=\left\{s_{\min }+k \Delta s, k=0,1, \ldots, K\right\}$. Aquí, la tasa mínima $s_{\min }$, el intervalo del interés $\Delta s$ y la cardinalidad $K+1$ son todos fijos. Por otro lado, se establece que $\mathcal{S}^{\prime}=\left\{s_{\min }+k \Delta s, k=\right.$ $0,1, \ldots, K-1\}$. Las probabilidades de transición del proceso $R$ son asignadas como sigue:

$$
p(x, y)=\left\{\begin{array}{c}
\frac{1}{3} s i(x, y) \in \mathcal{S}^{\prime} \times\{x, x \pm \Delta x\}, \\
\frac{1}{2} s i(x, y) \in\left\{s_{\text {min }} \times\left\{s_{\text {min }}, s_{\text {min }}+\Delta s\right\}\right\} \cup\left\{s_{\text {max }}\right\} \times\left\{s_{\text {max }}, s_{\text {max }}-\Delta s\right\}, \\
0 \text { en cualquier otro caso }
\end{array}\right.
$$

Esta función, junto con una tasa inicial de mercado $R(0)$, induce a una medida de probabilidad $\boldsymbol{P}_{\boldsymbol{R}(0)}$ en el espacio $\mathcal{S}^{T+1}$.

Volviendo al proceso de la tasa de deuda $r$, se asume que su valor inicial $r(0)$ coincide con la tasa de mercado $R(0)$ al mismo tiempo. En cualquier instante $t$, el deudor se enfrenta a la elección de continuar con la hipoteca de la deuda en pie, a tasa $r(t-1)$ o repagar la totalidad del capital debido, usando los beneficios de entrar en nueva hipoteca en las condiciones disponibles actuales expresadas por la tasa vigente del mercado $R(t)$. En este caso, decimos que la hipoteca se ha refinanciado.

Se estudia el caso donde se pueda ejercer esta posibilidad un número máximo de $N$ veces sobre un horizonte temporal $(0, \ldots, T-1)$. Si $N$ es igual al número de ajuste de veces $T-1$, la estrategia óptima para el prestatario es trivial: ejercer la opción cada vez que la tasa de mercado $R$ baje. Por lo tanto, hay que suponer que el número $N$ de oportunidades de refinanciación es estrictamente menor que el número de fechas. La tabla 2 indica los parámetros de entrada y los procesos dinámicos que definen las disposiciones del contrato.

Tabla 2. Parámetros de entrada y procesos dinámicos.

\begin{tabular}{lll}
\hline \multicolumn{1}{c}{ Cantidad } & Notación & Fórmula \\
\hline Deuda pendiente en $T_{0, T}$ & $B(t-1)$ & $\{0,1, \ldots, T\}$ \\
Rango de tasa de mercado & $S$ & $S_{\min }+k \Delta s, k$ \\
& & $=0,1, \ldots, K$ \\
Tasa de mercado inicial & $R(0)$ & Dada \\
Dinámica de la tasa de mercado & $R$ & $(R(t), t=0, \ldots, T)$ \\
Número de opciones & $n(0)$ & $N<T$ \\
Tasa inicial de deuda & $r(0)$ & $R(0)$ \\
Saldo deudor & $B(0)$ & 1 \\
& & \\
\hline
\end{tabular}

Fuente: Elaboración propia.

En cuanto a la decisión de refinanciación de la hipoteca, ésta puede ser descrita por un proceso $\alpha$, especificando en cada momento $t=0, \ldots, T-1$ si el deudor hipotecario continúa con su posición en las condiciones normales $\alpha=0$ o, si es posible, refinanciar su deuda $\alpha=1$. El proceso de control es descrito en la tabla 3. 
Tabla 3. Proceso de control.

\begin{tabular}{llc}
\hline \multicolumn{1}{c}{ Cantidad } & Notación & Fórmula \\
\hline $\begin{array}{l}\text { Proceso de política de } \\
\text { control }\end{array}$ & $n^{\alpha}$ & $(\alpha(t), t=0, \ldots, T-1)$ \\
$\begin{array}{l}\text { Dominio de la política de } \\
\text { control }\end{array}$ & $\mathcal{D}(\alpha(t))$ & $\{1\}$ si $\Sigma_{i=1}^{t} \alpha(i)<n(0)$ \\
& $\alpha(t)$ & $\begin{array}{l}0\} \text { en continuación } \\
\text { Política de control en } t\end{array}$ \\
& & 1 refinanciación
\end{tabular}

Fuente: Elaboración propia.

Por supuesto, la política de control elegida afecta a la dinámica de las variables de estado que caracterizan la posición del prestatario en el tiempo. Estas variables son: (1) el número $n^{\alpha}(t)$ de oportunidades de refinanciación disponibles dejadas para ejercitar en el futuro; (2) el tipo de interés corriente $r^{\alpha}(t)$; (3) el flujo de efectivo a plazos $P^{\alpha}(t)$ para el periodo corriente; (4) el resultado del saldo pendiente $B^{\alpha}(t)$. La tabla 4 proporciona todos los procesos sujetos a control. Está claro que la decisión de refinanciar o no en un momento dado t tiene efectos sobre $n^{\alpha}(t)$ y $\mathrm{r}^{\alpha}(\mathrm{t})$. El vínculo con las otras dos cantidades se aclara en la tabla 5.

Tabla 4. Sistema controlado.

\begin{tabular}{|c|c|c|}
\hline Cantidad & Notación & Fórmula \\
\hline $\begin{array}{l}\text { Proceso de opciones de } \\
\text { refinanciación }\end{array}$ & $n^{\alpha}$ & $\left(n^{\alpha}(t), t=0, \ldots, T-1\right)$ \\
\hline $\begin{array}{ll}\text { Oportunidades } & \text { de } \\
\text { refinanciación en } t & \end{array}$ & $n^{\alpha}(t)$ & \\
\hline $\begin{array}{l}\text { Proceso de la tasa de deuda } \\
\text { controlada }\end{array}$ & $r^{\alpha}$ & $\left(r^{\alpha}, t=0, \ldots, T-1\right)$ \\
\hline Tasa de deuda en $t$ & $r^{\alpha}(t)$ & $\begin{array}{l}R(t) \quad \text { si } \alpha(t)=1 \\
\left(r^{\alpha(t-1)}(t-1)\right. \\
\alpha(t)=0\end{array}$ \\
\hline Proceso de pago prorrateado & $P^{\alpha}$ & $\left(P^{\alpha}(t), t=0, \ldots, T-1\right)$ \\
\hline Proceso de deuda pendiente & $B^{\alpha}$ & $\left(B^{\alpha}(t), t=0, \ldots, T-1\right)$ \\
\hline
\end{tabular}

Fuente: Elaboración propia.

Tabla 5. Proceso de la deuda.

\begin{tabular}{ll}
\hline$B^{\alpha}(t)$ & Saldo de la deuda pendiente en $t$ \\
$B^{\prime}(t):=B^{\alpha}(t)\left(1+r^{\alpha}(t) / 52\right)$ & Capital más intereses sobre $[t, t+1]$ \\
$P^{\alpha}(t):=B^{\prime}(t) /(T-t)$ & Pro-rata del pago para [t, $t+1]$ \\
$B^{\alpha}(t+1):=B^{\prime}(t)-P^{\alpha}(t)$ & Saldo de la deuda pendiente en $t+1$ \\
\hline & Fuente: Elaboración propia.
\end{tabular}

Fuente: Elaboración propia.

El prestamista desea seleccionar una política de control que minimice el coste asociado con el sistema de amortización $\left(P^{\alpha}(t), t=0, \ldots, T-1\right)$. Con el propósito de ilustrar esta cuestión, consideramos la suma de todos estos pagos como una medida de este coste. Hay que tener en cuenta que no se considera el descuento para fines de evaluación, ni el coste de cambio de una hipoteca por otra, aunque más adelante (concretamente en el punto 3.3), se incluyen en el modelo los costes de transacción. El problema es, pues, calificado como sigue: 


$$
\min _{\alpha \in \mathcal{A}} E\left(\sum_{j=0}^{T-1} P^{\alpha}(j)\right)
$$

donde $\mathcal{A}$ denota la clase de políticas de control admisibles $\alpha=\left\{\alpha_{0}, \ldots, \alpha_{T-1}\right\}$ determinando el proceso de pago controlado $\left(P^{\alpha}(t), 0 \leq t<T\right)$. Específicamente, el proceso de repago $\left(\mathrm{P}^{\alpha}(\mathrm{t}), 0 \leq \mathrm{t}<\mathrm{T}\right)$ es definido sobre el espacio probabilístico $\left(\mathcal{S}^{\mathrm{T}}, \mathcal{B}\left(\mathcal{S}^{\mathrm{T}}\right), \mathrm{P}_{\mathrm{R}(0)}\right)$ y la expectativa se realiza bajo la probabilidad de medida $\mathrm{P}_{\mathrm{R}(0)}$ inducida en el espacio de las rutas por las probabilidades de transición XX y la condición inicial R(0).

Hay que tener en cuenta que cualquier política de control define una regla de parada multivariada $\left(\tau_{1}, \ldots, \tau_{n}\right), n \leq N$, que se define recursivamente por:

$$
\begin{aligned}
\tau_{1} & =\inf \{t \geq 0: \alpha(t)=1\} . \\
\tau_{k+1} & =\inf \left\{t>\tau_{k}: \alpha(t)=1\right\} .
\end{aligned}
$$

La tarea es construir un modelo para describir la estructura financiera mostrada arriba, a continuación, desarrollar un algoritmo obteniendo el valor mínimo en (3) y finalmente determinar la correspondiente política de control óptima:

$$
\alpha^{*}=\left(\alpha^{*}(0), \ldots, \alpha^{*}(T-1)\right)
$$

Este último es equivalente a la regla óptima de parada multivariada $\left(\tau_{1}^{*}, \ldots, \tau_{N}^{*}\right)$. Terminamos esta sección derivando una expresión para el saldo pendiente en $t+1$ en términos de la política de control adoptadas y el nivel de endeudamiento registrado un intervalo de tiempo antes:

$$
B(t+1)=B^{\prime}(t)-P(t)=B^{\prime}(t)-\frac{B^{\prime}(t)}{T-t}=c\left(r^{\alpha(t)}(t)\right) B(t) \mathcal{T}(t)
$$

Donde $c(x)=1+x / 52$ denota el coeficiente de acumulación que corresponde a la tasa $x \mathrm{y}$ $\mathcal{T}(t)$ es el coeficiente de prorrateo definido por $1-1 /(T-t)$.

\section{Implantación y algoritmo.}

\subsection{Políticas de control de Markov.}

Cabe recordar que la variable de estado de un problema de control de Markov se define como la información en la que la política de control se elige en cualquier momento. Con más precisión, éste es el conjunto de variables observables sobre la cual $\alpha(t)$ pueden ser determinada en un momento dado en el tiempo.

Se observa que el tiempo $t$ de equilibrio, $B(t)$, y la tasa de deuda, $r(t)$, determinan por completo tanto la actualización del capital más interés $B^{\prime}(t)$ y el esquema de pago en cuotas constantes $P(t)$, que a su vez entra en el objetivo funcional visto en (3). Puesto que el objetivo se ve afectado tanto por $B(t)$ y $r(t)$, el tiempo t de control $\alpha(t)$ debería depender de estas variables. La dependencia sobre $B(t)$ es sencilla. Si cualquier oportunidad de refinanciación es aún posible, por ejemplo, $0<n(t) \leq N$ entonces la tasa de la deuda es fijada a la mejor entre su valor previo $r(t-1)$ y la tasa corriente de mercado $R(t)$ observada al mismo tiempo en dicho mercado. Si, en el caso contrario, todas las oportunidades de refinanciación se han agotado, por ejemplo, $n(t)=$ 0 , entonces, la nueva tasa de deuda corriente $r(t)$ debe concordar con su valor previo $r(t-1)$. En general, $r(t)$ y por lo tanto, la política de control de Markov $\alpha(t)$, depende de $(R(t), r(t-1), n(t))$. 
Estas premisas nos llevan a considerar políticas de control cuyo valor de tiempo $t$ depende del saldo pendiente $B(t)$, la tasa de mercado $R(t)$, la tasa de la deuda a un periodo vista $r(t-1)$ y el número $n(t)$ de opciones disponibles de refinanciación:

$$
\alpha(t)=F(t,(B(t), R(t), r(t-1), n(t))) .
$$

Si $B, R, r^{-}, n$, representan posibles valores tomados por $B(t), R(t), r(t)$ y $n(t)$, respectivamente, el cuarteto $\left(B, R, r^{-}, n\right)$ es una variable estado candidata.

Se adopta el principio de programación dinámica para los fines del cómputo de la función valor y la política de ejercicio óptimo durante la vida del contrato. Desde que la variable estado $B(t)$ es continua, se evita una aplicación directa de este principio. Sin embargo, la función valor es homogénea de grado uno en esta variable y la función valor se puede escribir como:

$$
V(t,(y, x, r, n)):=y \times V^{*}(t,(x, r, n)) .
$$

Es decir, se refiere a $V^{*}$ como la "Función Valor Unitaria" porque representa la función valor por unidad de saldo. Puesto que $y$ es no-negativa, la política óptima para $V$ coincide con la política óptima para $V^{*}$. Como consecuencia de ello, las reglas de parada admisibles son independientes de $y$. Por lo tanto, se consideran las variables de control de la forma:

$$
\alpha(t)=F(t,(R(t), r(t-1), n(t))) .
$$

La variable de control así definida es $\mathcal{F}_{t}$-medible, implicando que la política de control (6) es admisible. La variable estado de nuestro problema es $\left(R, r^{-}, n\right)$, definida en un sub-conjunto de $\mathcal{H}=\mathcal{S} \times \mathcal{S} \times\{0, \ldots, N\}$. Por ejemplo, si $t=1, r \in\left\{r_{0}, r_{0}+\Delta s, r_{0}-\Delta s\right\} \subset \mathcal{S}$. Resulta que la pérdida en términos de complejidad de cálculo para restringir los cálculos numéricos para el dominio exacto de la variable estado superan la ganancia resultante de la reducción del número de cálculos. En consecuencia, se decide saltar, considerando las limitaciones del dominio, mientras se realiza la optimización del algoritmo y se calcula la función valor en todo el dominio $\mathcal{H}$.

\subsection{Algoritmo de programación dinámica.}

Habiendo identificado la variable estado, el siguiente paso es el cálculo recursivo de la función valor $V^{*}$ y la determinación de la estrategia de refinanciación óptima consistente en el $N$-ésimo tiempo de parada estrictamente creciente de $\mathcal{T}$-valorada.

\section{- Función Valor en $T$ :}

En el momento $T$, el deudor paga el capital vivo $T-1$ más los intereses devengados entre $T-1$ y $T$. Entonces el contrato se extingue y se vuelve

para todo $y, x, r, n$ admisibles.

$$
V(T,(x, y, r, n))=0,
$$

\section{- Función Valor en $T$ - 1:}

Si ninguna opción de refinanciación está, ya, disponible, $p . e j .: n=0$, el deudor incurre en un pago igual a $T$ - 1, una deuda pendiente, $y$, más los intereses devengados entre $T-1$ y $T$ de acuerdo a la tasa $r$, que es

$$
C N R^{0}(T-1, y, x, r)=y \times c(r) \times \mathcal{T}^{*}(T-1),
$$


Donde $c(r):=(1+r / 52), \mathcal{T}^{*}(t):=1 /(T-t)$ es el complemento al coeficiente $\mathcal{T}(t)$ a la unidad. Si alguna opción de refinanciación sigue estando disponible, es decir $n>0$, se compara con el coste $C R$ resultante de la refinanciación, es decir, $\alpha=1$ con el CNR, coste resultante de continuar en las condiciones establecidas, es decir $\alpha=0$. La primera es la suma de los intereses $C P_{[T-1, T]}(y, x)$ acumulados en la deuda pendiente y sobre el periodo entre $T-1$ y $T$ de acuerdo a la nueva tasa de mercado $x$ y a la nueva función de descuento de valor esperado $D E C(T, y, x, r, n-$ 1) calculada un periodo en el tiempo adelante, es decir, en $(T-1)+1=T$, correspondiente a una oportunidad menos de refinanciación, que es:

$$
\begin{aligned}
C N R(T-1, y, x, r) & =C P_{[T-1, T]}(y, x)+D E C(T, y, x, r, n-1) \\
& =y \times c(r) \times \mathcal{T}^{*}(T-1)+0
\end{aligned}
$$

Este último es definido de manera similar, excepto por la nueva tasa de deuda que ahora es igual a la tasa de la deuda actual $r$ :

$$
\begin{aligned}
C N R(T-1, y, x, r) & =C P_{[T-1, T]}(y, x)+D E C(T, y, x, r, n) \\
& =y \times C(r) \times \mathcal{T}^{*}(T-1)+0
\end{aligned}
$$

El valor de la función $T-1$ es pues:

$$
\begin{aligned}
& V(T-1,(y, x, r, n)) \\
= & \begin{cases}\min \{C R(T-1, y, x, r, n), C N R(T-1, y, x, r, n)\}, & n \geq 1 \\
C N R^{0}(T-1, y, x, r), & n=0\end{cases} \\
= & \begin{cases}\min \left\{y \times c(x) \mathcal{T}^{*}(T-1), y \times c(r) \mathcal{T}^{*}(T-1)\right\}, & n \geq 1 \\
y c(r) \mathcal{T}^{*}(T-1), & n=0\end{cases}
\end{aligned}
$$

Desde que $c\left(\right.$ ) es una función creciente y ambos y $\mathcal{T}^{*}(T-1)$ son positivos, si $n>0$, la decisión óptima es refinanciar la hipoteca, siempre que la nueva tasa de mercado $x$ sea inferior a la tasa de deuda pendiente $r$. La política de control óptima en $T-1$ es:

$$
\begin{array}{r}
\alpha(T-1,(y, x, r, n))=\alpha(T-1)(x, r, n) \\
= \begin{cases}1 & \text { si } n \geq 0 y x<r \\
0 & \text { si } n=0 y r \leq x\end{cases}
\end{array}
$$

\section{- Función Valor en $t$}

El valor de la función en $t$ es calculado como en la fórmula 7 pero reemplazando $T$-1 por $t$ :

$$
\begin{aligned}
& V(t,(y, x, r, n)) \\
= & \begin{cases}\min \{C R(t, y, x, r, n), C N R(t, y, x, r, n)\}, & n \geq 1 \\
C N R^{0}(t, y, x, r), & n=0\end{cases}
\end{aligned}
$$

La diferencia principal aquí es que se necesitan cálculos más intensivos:

$$
\begin{aligned}
C N R(t, y, x, r) & =C P_{[t, t+1]}(y, x)+D E C(t+1, y, x, r, n-1) \\
& =y c(x) \times \mathcal{T}^{*}(t)+E^{P_{x}}(V(t,(B(t+1), x, n-1))),
\end{aligned}
$$


Donde $\mathcal{T}^{*}:=1 /(T-t)$ es el complemento a uno de los coeficientes $\mathcal{T}(t)$ y el símbolo “.” indica el argumento con respecto a qué expectativa va a ser calculada. Se ha de tener en cuenta que (a), el equilibrio en $t+1$ es igual a $B(t+1)=c(r(t) B(t) \mathcal{T}(t)$, (b), el valor esperado es calculado sobre los posibles valores tomados por la tasa de mercado $R(t+1)$ y (c), los factores $V$ en el producto $y \times V^{*}$, coste de refinanciación, se vuelven:

$$
\begin{aligned}
C R & =y c(x) \mathcal{T}^{*}(t)+E^{P_{x}}(V(t+1,(c(x) y \mathcal{T}(t), R(t+1),(\cdot), x, n-1))) \\
& \left.=y c(x) \mathcal{T}^{*}(t)+c(x) \mathcal{T}(t) y E^{P_{x}}\left(V^{*}(t+1),(R(t+1)(\cdot), x, n-1)\right)\right)
\end{aligned}
$$

Cálculos análogos para el coste de continuación llevan a:

$$
\begin{aligned}
C N R= & C P_{[t, t+1]}(y, r)+D E C(t+1, y, x, r, n-1) \\
= & y c(r) \mathcal{T}^{*}(t)+E^{P_{x}}(V(t+1,(B(t+1), \cdot, r, n))) \\
& \left.=y c(r) \mathcal{T}^{*}(t)+c(r) \mathcal{T}(t) y E^{P_{x}}\left(V^{*}(t+1),(R(t+1)(\cdot), r, n)\right)\right) .
\end{aligned}
$$

El mismo coste de continuación cuando todas las opciones han sido ejercidas, es decir, $n$ $=0$, es

$$
C N R^{0}(t, y, x, r)=\operatorname{CNR}(t, y, x, r, 0) .
$$

Mediante la recopilación de todas estas expresiones y dividiendo por el saldo pendiente $y$, se da la aproximación a una fórmula para el tiempo $t$ de la función de valor unitaria:

$$
V^{*}=\left\{\begin{array}{cc}
\min \left(c(x) \mathcal{T}^{*}(t)+\right. & \\
\left.+c(x) \mathcal{T}(t) E^{P_{x}}\left(V^{*}(t+1),(R(t+1)(\cdot), x, n-1)\right)\right), & \\
\left.c(r) \mathcal{T}^{*}(t)+c(r) \mathcal{T}(t) E^{P_{x}}\left(V^{*}(t+1),(R(t+1)(\cdot), x, n-1)\right)\right), & \text { si } n \geq 1, \\
\left.c(r) \mathcal{T}^{*}(t)+c(r) \mathcal{T}(t) E^{P_{x}}\left(V^{*}(t+1),(R(t+1)(\cdot), r, 0)\right)\right), & \text { si } n=0 .
\end{array}\right.
$$

La regla de parada óptima en $t$ no es tan simple como en la fórmula (8) ya que se necesita minimización explícita para decidir si financiar de nuevo o no. Un último comentario es que la probabilidad bajo la cual se toman las expectativas está determinada por la fórmula del punto 2.2 (hipoteca con tipo flexible), a través de la regla $P_{x}(\{y\}):=P(x, y)$ en todos los casos.

\section{- Función Valor en 0}

En $t=1$, la función de valor unitario $V^{*}$ se convierte en independiente de las variables $r$ y $n$. De hecho, ésta es la primera vez en la que al deudor se le permite refinanciar y todas las opciones $N$ están disponibles. Hay que tener en cuenta que la tasa de la deuda en $[0,1]$ es $r_{0}$ y $R(1) \in\left\{r_{0}, r_{0}+\Delta s, r_{0}-\Delta s\right\}$. La función valor es dada por $V=V^{*}\left(0,\left(r_{0}, r_{0}, N\right)\right)=$ $c\left(r_{0}\right) \mathcal{T}^{*}(0)+c\left(r_{0}\right) \mathcal{T}^{*}(0) \times E^{P_{r_{0}}}\left(V^{*}\left(1,\left(\cdot, r_{0}, N\right)\right)\right)$. Este es el precio justo del contrato de hipoteca en el momento 0 .

\subsection{Costes de transacción.}

Para proporcionar un incentivo eficaz, muchas hipotecas prescriben una cuota para solicitar un pago por anticipado: el deudor hipotecado que desea extinguir el contrato está obligado a pagar un coste adicional que generalmente oscila entre 0.5 y 2 puntos porcentuales sobre el capital pendiente. Para incluir esta función en el modelo, es necesaria una pequeña modificación. Una vez más, la función valor se factoriza como:

$$
V(t,(y, x, r, n)):=y \cdot V^{*}(t,(x, r, n))
$$


Desde que los costes de transacción son proporcionales a los saldos pendientes pueden ser incluidos en la función valor como una constante añadiéndose a la función $C R$. Sea $\varphi$ una representación de la cuota del saldo pendiente que se destaca como una tarifa pre-pago. En general, la función valor en $t V^{*}=V^{*}(t,(x, r, n))$ por unidad de equilibrio se calcula como:

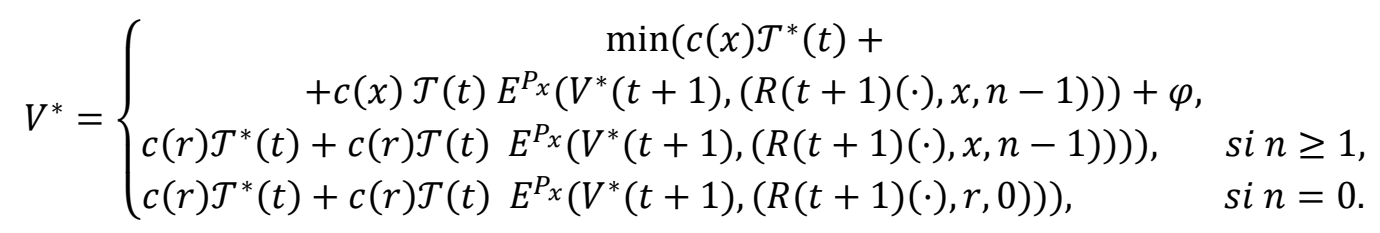

\section{Resultados.}

La implantación y simulación del modelo expuesto anteriormente se ha hecho usando Matlab®. Para ello se ha calculado el valor justo del contrato de hipoteca $V\left(0,\left(1, r_{0}, N\right)\right)$ correspondiente a una tasa $r_{0}=3.5 \%$ con un número de oportunidades de refinanciación $N=4$. Éste es el coste mínimo para el deudor hipotecario, es decir, la resultante de la política de control óptimo, obteniendo un coste óptimo igual a 1.4404 .

La política de refinanciación óptima está expresada como una regla de transformación de cualquier posible configuración de la variable de estado en una acción que consiste en continuar o refinanciar la hipoteca. Para tener una idea concreta acerca de la política óptima, se traza una muestra del proceso de la tasa de mercado e indicamos los cuatro tiempos óptimos de refinanciación (figura 1).

Figura 1. Ejemplo de trayectoria de la tasa de interés de mercado.

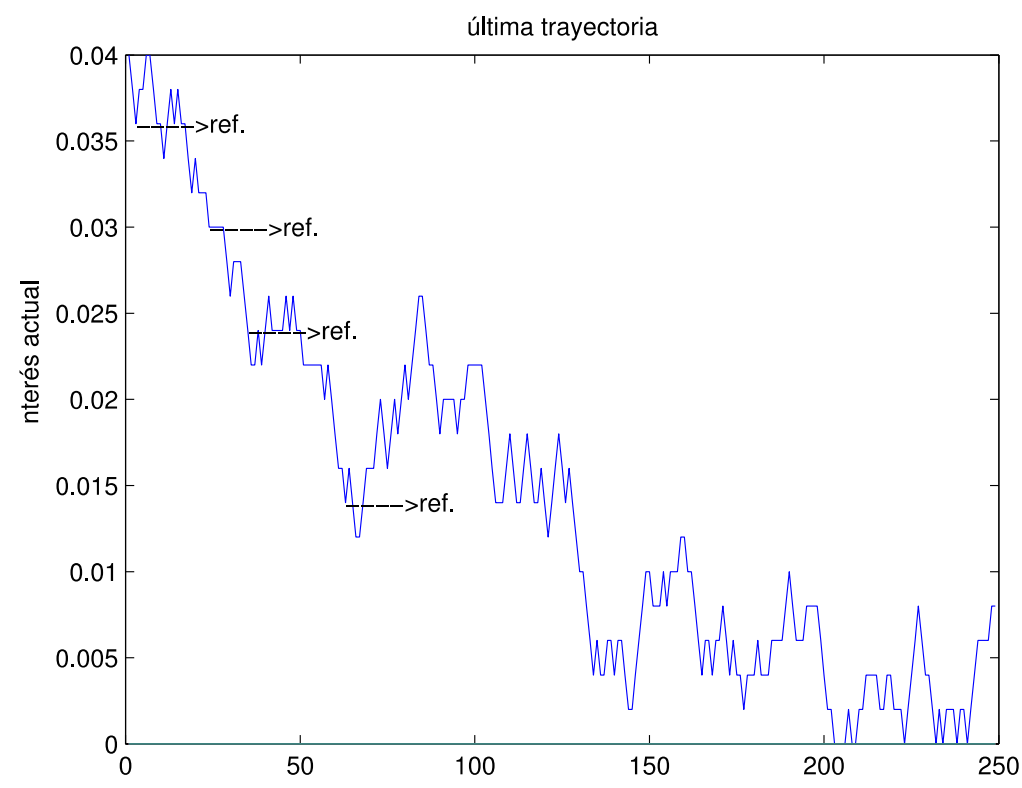

La expresión “-> ref” indica los 4 tiempos óptimos de refinanciación.

Fuente: Elaboración propia.

Se puede observar que, para los parámetros simulados, todas las opciones de refinanciación son ejercidas en las primeras 50 semanas, muy poco tiempo en comparación con el horizonte de amortización. Mientras que el tipo de interés de mercado incrementa, ninguna refinanciación tiene 
lugar. En las semanas posteriores a la última refinanciación, alrededor de la semana 60, las tasas de mercado crecen para luego, volver a decrecer alcanzando en alguna ocasión el 0.

Una imagen más clara de la política de control óptimo se obtiene mediante el cálculo de las distribuciones de los cuatro tiempos óptimos de refinanciación. Las expresiones analíticas para estas distribuciones no están disponibles. Sin embargo, se puede calcular una estimación de la muestra de su forma mediante la simulación de un gran número de trayectorias, a continuación, guardar los correspondientes tiempos óptimos de parada y, finalmente, calcular el histograma de frecuencias relativas de los cuatro tiempos de refinanciación. La figura 2 muestra una distribución de probabilidad de la muestra para las dos primeras veces de refinanciación optimas, obtenida a través de 10000 muestras del proceso de la tasa de mercado. Esta distribución se ajusta a una de tipo gamma, aunque depende, en este caso, del proceso de simulación seguido.

La primera opción tiende a ser ejercida dentro de las dos primeras semanas, mientras que la segunda parada parece abarcar un periodo de tiempo más amplio. Es interesante notar que una refinanciación de la opción no se ejerce durante el primer período. La primera refinanciación muestra una distribución de punta, con un máximo alcanzado en $t=2$. Parece que, si la tasa de mercado disminuye durante el primer periodo, la alta proximidad al periodo inicial tiende a compensar la ventaja derivada de la refinanciación. Por el contrario, si la tasa de mercado decrece en el segundo periodo, este efecto se invierte y la hipoteca se refinancia.

Figura 2. Muestra de la distribución de probabilidad de las dos primeras refinanciaciones sobre $\mathbf{n}=10000$ muestras.

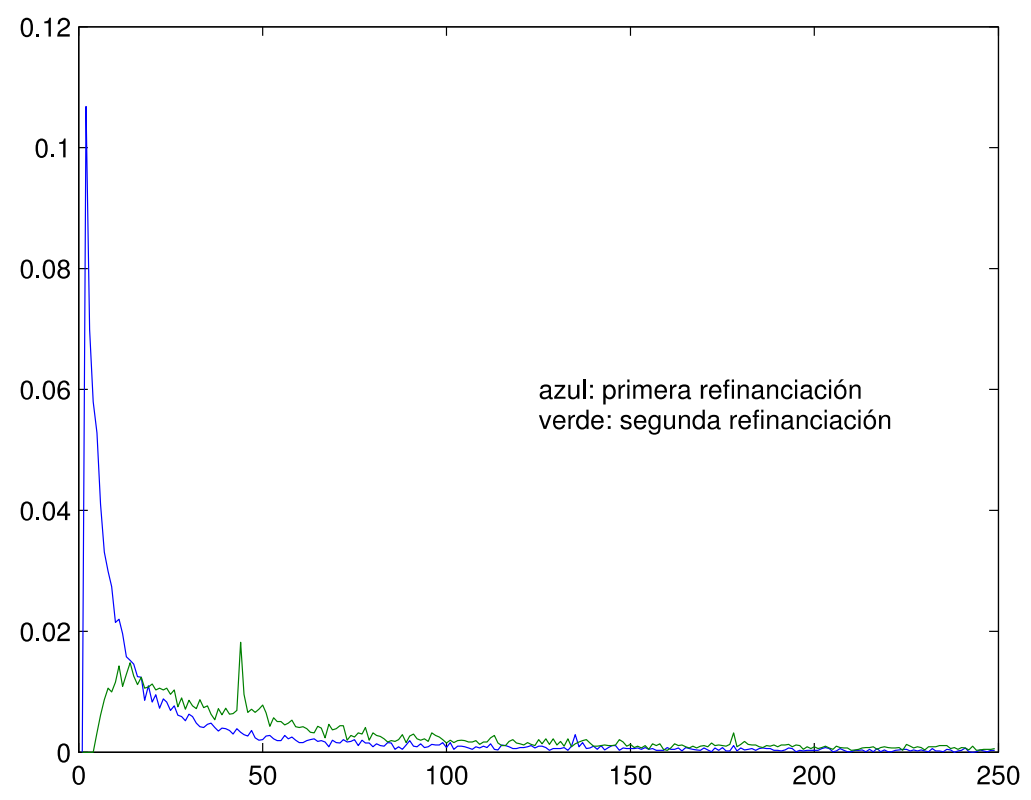

Fuente: Elaboración propia.

La figura 3 muestra las dos últimas refinanciaciones, donde se observa que estos tiempos de refinanciación se agrupan en el extremo más alejado del horizonte temporal; sin embargo, también muestran un comportamiento con picos muy marcados. 


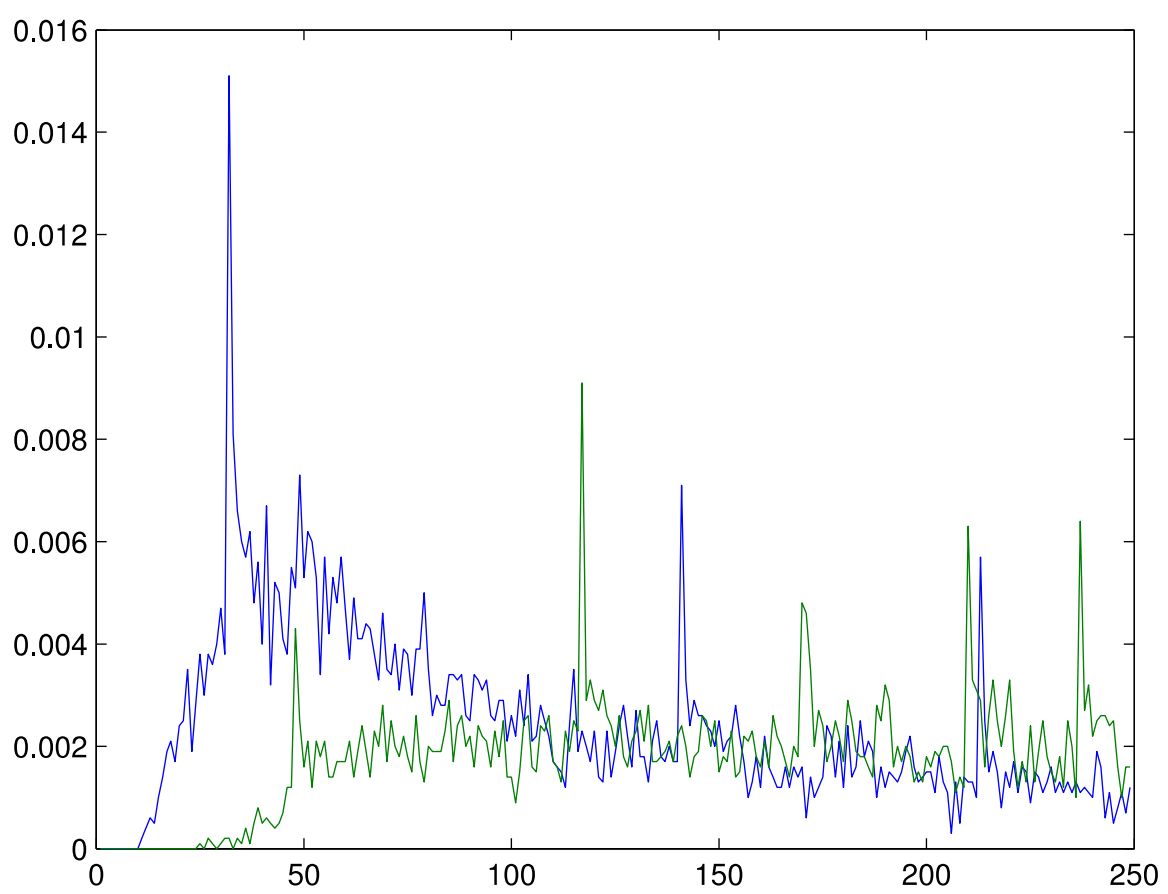

Fuente: Elaboración propia.

\section{Conclusiones.}

El desarrollo de un modelo de refinanciación de una hipoteca de este tipo puede ser interesante porque es un caso bastante común hoy día el que un prestatario quiera refinanciar su hipoteca dadas las condiciones actuales. Además, se trata de una simulación que al estar basada únicamente en conceptos matemáticos y posterior desarrollo con el software pertinente no hemos tenido que recurrir a ninguna base de datos, hecho que, por otro lado, planteó una dificultad elevada el conseguir datos reales del mercado.

El objetivo de dicha simulación era obtener la política de refinanciación óptima dadas unas condiciones de partida, dicha política, como se ha dejado claro anteriormente estaba representada como una regla de transformación de la variable de estado en una acción que consiste en continuar con la hipoteca actual o refinanciarla, estando fijadas las oportunidades de refinanciación en 4. Como resultado, para esas 4 oportunidades de refinanciación, se ejercen todas las opciones de refinanciación en las primeras 50 semanas y queda claro que tanto elevadas tasas de entrada como altas tarifas tienen un efecto negativo en el mínimo coste sostenible por el hipotecado. Es importante mencionar aquí que el modelo representa una opción de refinanciación simple, sin tener en cuenta otros elementos relevantes a la hora de considerar una refinanciación, como hacen Tai y Przasnyski (2000), donde incluyen factores como la reducción en las cuotas, el periodo de espera para el nuevo préstamo (que suele haber unos días hasta que se concede donde se generarían ciertos intereses), el coste de oportunidad de la refinanciación o el tipo de interés marginal del deudor.

La cuestión ahora es si las proposiciones de Basilea III influirán de forma significativa en el sentido de ayudar a que las probabilidades de otra crisis como la del 2008, sean ampliamente 
reducidas (Walter, 2010; Hannoun, 2010). A priori, dicho acuerdo contiene reformas bastante útiles: apoyo a un ratio de apalancamiento, un regulador de capital y la propuesta de tratar con la pro-ciclicalidad a través de provisiones dinámicas basadas en las pérdidas esperadas. Sin embargo, también se demanda más implicación de los agentes sociales, de los gobiernos de cada país con un sistema financiero estable en el sentido de que ayuden a los más inestables, que aporten sus conocimientos para asegurar la solidez financiera de un sistema bastante mermado en calidad y credibilidad actualmente, que se persiga cualquier conducta sombría de omisión u ocultación de información y se penalice, regulándose más aún, cualquier actividad financiera y acentuando los sistemas de planificación, control y supervisión en todos los países, sobre todo aquellos más expuestos a cualquier tipo de riesgo.

Investigaciones de autores como Kalotay, Yang y Fabozzi (2008), proponen aplicar herramientas de valoración de bonos para la gestión del riesgo del hogar. Otros como Sy (2008) desarrollan una corriente basada en los modelos causales para evitar los defectos de los actuales modelos de crédito. Y más recientemente, Rasmussen, Madsen y Poulsen (2014) plantean un modelo basado en programación estocástica para la elección de hipotecas y aplicándolo al mercado hipotecario danés, comparan el rendimiento con un punto de referencia, que no es otro que la simple "regla de oro", basada en la práctica por la que se rigen los bancos daneses a la hora de aconsejar a los hipotecados. Es por ello que solo con el entendimiento de la causalidad del proceso de impago del crédito podremos construir modelos deductivos según señalan Capuano, (2008) y Campbell (2012), o las recientes innovaciones en este campo como son los modelos de correlaciones de impago usando modelos cópula que sean capaces de hacer predicciones en un entorno y una estructura de mercado cambiantes en un contexto donde se demanda un sistema financiero de vivienda eficiente y estable.

\section{Referencias}

Campbell, J.Y. (2012). Mortgage Market Design, NBER Working Paper, nº w1939.

Capuano, C. (2008). Recent Advances in Credit Risk Modelling, IMF Working Paper.

Hannoun, H. (2010). The Basel III Capital Framework: A Decisive Breakthrough. BoJ-BIS high Level Seminar on "Financial Regulatory Reform: Implications for Asia and the Pacific", Hong Kong SAR, 22 November 2010.

Kalotay, A.J., Yang, D., \& Fabozzi, F.J. (2008). Optimal Mortgage Refinancing: Application of Bond Valuation Tools to Household Risk Management. Applied Financial Econometric Letters, 4, 141-149.

Longstaff, F.A. (2005). Optimal Recursive Refinancing and the Valuation of Mortgage-Backed Securities. Real Estate Economics, 33(4), 619-661.

Pliska, S.R. (2006). Mortgage Valuation and Optimal Refinancing. Stochastic Finance, Springer.

Rasmussen, K.M., Madsen, C.A., \& Poulsen, R. (2014). Can Home Owners Benefit from Stochastic Programming Models? - A Study of Mortgage Choice in Denmark. Computational Management Science, 11(1), 5-23.

Roncoroni, A., \& Moro, A. (2006). Flexible-Rate Mortgages. International Journal of Business, 11(2), 143-157.

Roncoroni, A., \& Fusai, G. (2008). Implementing Models in Quantitative Finance: Methods and 
Cases, $2^{\text {nd }}$ ed, Springer.

Sy, W. (2008). Credit Risk Models: Why They Failed in Credit Crisis. Australian Prudential Regulation Authority (Sydney).

Tai, L.S., \& Przasnyski, Z. H. (2000). Evaluating Refinancing Strategies Precisely. Journal of Financial and Strategic Decisions, 13(3), 9-21.

Walter, S. (2010). Basel III and Financial Stability. 5th Biennial Conference on Risk Management and Supervision. Financial Stability Institute, Bank for International Settlements, Basel, 3-4 November 2010. 